\begin{tabular}{|c|l|}
\hline Title & A mphipathic al pha helix mediates the heterodimerization of soluble guanylyl cyclase. \\
\hline Author(s) & Shiga, Takumi; Suzuki, Norio \\
\hline Citation & $\begin{array}{l}\text { Zoological Science, 22(7), 735-742 } \\
\text { https://loi.org/10.2108/2s. 22.735 }\end{array}$ \\
\hline Issue Date & 2005-07 \\
\hline Doc URL & http://hdl.handle.net/2115/14186 \\
\hline Type & article(author version) \\
\hline File Information & shiga.pdf \\
\hline
\end{tabular}

Instructions for use 


\section{Amphipathic $\alpha$-Helix Mediates the Heterodimerization of Soluble Guanylyl Cyclase}

\section{Takumi Shiga and Norio Suzuki*}

Division of Biological Sciences, Graduate School of Science, Hokkaido University, Sapporo 060-0810, Japan

Short title: Heterodimerization of Soluble GC

*Corresponding author. Tel.: +81-11-706-4908; Fax: +81-11-706-4461.

E-mail address: norio-s@sci.hokudai.ac.jp 
ABSTRACT - Soluble guanylyl cyclase (soluble GC) is an enzyme consisting of $\alpha$ and $\beta$ subunits and catalyzes the conversion of GTP to cGMP. The formation of heterodimer $\left(\alpha_{1} / \beta_{1}\right.$ or $\left.\alpha_{2} / \beta_{1}\right)$ is essential for the catalytic activity. Each subunit has been shown to comprise three functionally different parts: a C-terminal catalytic domain, a central dimerization domain, and an N-terminal regulatory domain. The central dimerization domain in the $\beta_{1}$ subunit, which contains an N-terminal binding site (NBS) and a C-terminal binding site (CBS), have been postulated to be responsible for the formation of $\alpha / \beta$ heterodimers. In this study, we analyzed heterodimerization by pull down assay using the affinity between histidine tag and $\mathrm{Ni}^{2+}$ Sepharose after co-expression of various $\mathrm{N}$ - and C-terminally truncated $\alpha_{1}$ mutants (FLAG tag) and $\beta_{1}$ wild type (histidine tag) in the baculovirus/Sf9 system, demonstrating that the CBS-like sequence of the $\alpha_{1}$ subunit is critical for the formation heterodimer with the $\beta_{1}$ subunit and the NBS-like sequence of the $\alpha_{1}$ subunit is essential for the formation of enzymatically active heterodimer, although itself was not involved in heterodimerization. The analysis of the secondary structure of the $\alpha 1$ subunit predicted the existence of an amphipathic $\alpha$-helix in the residues 431-464. Experiments with site-directed $\alpha_{1}$ subunit mutant proteins demonstrated that the amphipathicity of the $\alpha$-helix is important for the formation of a correct active center in the dimer.

Key words: soluble guanylyl cyclase, cGMP, heterodimer, dimerization domain, recombinant protein, pull down assay, amphipathic $\alpha$-helix, secondary structure 


\section{INTRODUCTION}

Both the soluble and membrane forms of guanylyl cyclases (GCs) catalyze the conversion of GTP to cGMP, which is a ubiquitous second messenger in intracellular signaling cascades and responsible for a wide variety of physiological responses (Drewett and Garbers, 1994; Garbers and Lowe, 1994; Garbers et al., 1994). Soluble GC is a heterodimer consisting of $\alpha$ and $\beta$ subunits, and contains a prosthetic ferrous heme group to which nitric oxide (NO) binds with high affinity. Formation of an NO-heme complex and a subsequent conformational change of the heterodimer are responsible for the up to 200-fold increases in the catalytic rate (Gerzer et al., 1981; Humbert et al., 1990; Stone and Marletta, 1994). In mammals, cDNAs for four soluble GC subunits $\left(\alpha_{1}, \alpha_{2}, \beta_{1}\right.$, and $\left.\beta_{2}\right)$ have been isolated from various tissues (Koesling et al., 1988; Koesling et al., 1990; Yuen et al., 1990; Harteneck et al., 1991). Both the $\alpha_{1} / \beta_{1}$ and $\alpha_{2} / \beta_{1}$ heterodimers have been shown to be enzymatically active when formed in an in vitro expression system (Russwurm et al., 1991). A single expression of either one of the subunits has been shown to yield an $\alpha_{1} / \alpha_{1}$ - or a $\beta_{1} / \beta_{1}$-homodimer, although both homodimers were catalytically inactive (Zabel et al., 1999). In previous studies, we have isolated and characterized the cDNA and genomic DNA clones encoding the soluble GC subunits, OlGCS- $\alpha_{1}$ and OlGCS- $\beta_{1}$, both of which are aligned in tandem on the genome of the medaka fish Oryzias latipes (Mikami et al., 1998, Mikami et al., 1999). We recently demonstrated that the medaka fish contains another type of a soluble GC subunit gene, OIGCS- $\alpha_{2}$, which locates on different linkage group of OlGCS- $\alpha_{1}$ and OIGCS- $\beta_{1}$ (Yao et al., 2003).

Each subunit of soluble GC can be divided functionally into three parts: a C-terminal catalytic domain, a central region commonly referred to as the dimerization 
domain, and an N-terminal regulatory domain participating in heme-binding (Koesling, 1999). The His ${ }^{105}$ of the $\beta_{1}$ subunit has been identified as the proximal heme ligand site (Wedel et al., 1994; Zhao et al., 1998; Foerster et al., 1996). The C-terminal catalytic domain is highly conserved among various soluble GC subunits and is assigned as being responsible for catalysis based on homology to the related domain of adenylyl cyclase (Koesling, 1999). Dimerization has been attributed to the central region of the soluble GC subunits mainly based on studies with the peptide receptor/membrane GCs (Wilson and Chinkers, 1996). Recently, it has been demonstrated that the dimerization region of the $\beta_{1}$-subunit consists of 205 amino acid residues over the regulatory and central regions and contains a discontinuous site of 41 amino acid residues (N-terminal binding site: NBS) and 30 amino acid residues (C-terminal binding site: CBS), respectively, facilitating binding of the $\beta_{1}$ subunit to the $\alpha_{1}$ subunit (Zhou et al., 2004). On the other hand, the function of the N-terminal part of the $\alpha_{1}$ subunit has been remained to be solved, although there are several papers to demonstrate that the $\mathrm{N}$-terminal part of the $\alpha_{1}$ subunit is critical for heme-binding (Wedel et al., 1995) and that deletion of the N-terminal 259 amino acids from the $\alpha_{1}$ subunit had no effect on the properties of the enzyme at all, and the amino acids 259-364 of the $\alpha_{1}$ subunit represent an important functional domain for the transduction of the NO-activation signal (Koglin and Behrends, 2003). Although soluble GC has not been crystallized yet for X-ray diffraction analysis, it has been predicted that its catalytic domain is resemble to that of the class III adenylyl cyclases (Tucker et al, 1998; Sunahara et al., 1998). Structural analysis of the catalytic core of the adenylyl cyclases demonstrated an antiparallel orientation of the two catalytic domains $\left(\mathrm{C}_{1}\right.$ and $\mathrm{C}_{2}$ ) with two pockets, each being formed by both the catalytic domains (Zhang et al., 1997; Tesmer et al., 1997). 
To investigate the dimerization region of the $\alpha_{1}$ subunit, we generated several recombinant soluble GC subunits in an expression system with Sf9 cells and performed pull down assay using the affinity between histidine tag and $\mathrm{Ni}^{2+}$ Sepharose. Here, we report that the CBS-like sequence of OlGCS- $\alpha_{1}$ is involved in the formation of heterodimer with OlGCS- $\beta_{1}$ and that the amphipathic $\alpha$-helix of OlGCS- $\alpha_{1}$ is critical for the formation of heterodimer with OlGCS- $\beta_{1}$. We also describe that the NBS-like sequence of OlGCS- $\alpha_{1}$ plays an important role in the formation of an enzymatically active heterodimer, although it is not involved in the formation of heterodimer with OlGCS- $\beta_{1}$.

\section{MATERIALS AND METHODS}

\section{Construction of plasmid for histidine-tagged OIGCS- $\beta_{1}$-full length protein (FL $\left.{ }_{H T}\right)$}

Total RNA was isolated from the adult brain of $O$. latipes by the acid/guanidinium/thiocyanate/phenol/chloroform extraction method (Chomczynski and Sacchi, 1987). Total RNA (5 $\mu \mathrm{g})$ was used as the template to synthesize the first strand cDNA using an oligo(dT) primer and Super Script II preamplification system for the first-strand cDNA synthesis (Gibco BRL, Tokyo, Japan) according to the manufacturer's protocol. To obtain a C-terminal histidine-tagged OlGCS- $\beta_{1}$-full length protein (OlGCS- $\left.\beta_{1}-\mathrm{FL}_{\mathrm{HT}}\right)$, we designed the following primers: 5'-GTG GAT CCA TGT ATG GTT TTG TGA AT-3' and 5'-CGC GTC GAC TTA GTG ATG GTG ATG GTG ATG TGC CTT GTC AGC GTC GCT GC-3’’ PCR was performed using Ex Taq polymerase (TaKaRa, Otsu, Japan) according to manufacturer's protocol with the following PCR conditions: 30 cycles of $30 \mathrm{sec}$ at $94^{\circ} \mathrm{C}, 30 \mathrm{sec}$ at $50^{\circ} \mathrm{C}, 4 \mathrm{~min}$ at $72^{\circ} \mathrm{C}$, and a final extension of $5 \mathrm{~min}$ at $72^{\circ} \mathrm{C}$. The cDNA fragment amplified by the 
PCR was purified and subcloned into the plasmid vector pBluescript II KS(-) (Stratagene, La Jolla, California, USA). A BamHI/SalI insert from the OIGCS- $\beta_{1}$ clone in pBluescript II KS(-) vector was ligated into BamHI/SalI cut pFASTBAC1 vector (Invitrogen Japan K.K., Tokyo, Japan).

\section{Construction of plasmids for FLAG-tagged OIGCS- $\alpha_{1}$-full length protein (FLFLAG)} and its truncated mutant proteins

To obtain a C-terminal FLAG-tagged OlGCS- $\alpha_{1}$-full length protein (OlGCS- $\alpha_{1}-\mathrm{FL}_{\mathrm{FLAG}}$ ), several N-terminal and C-terminal deletion mutant proteins of OlGCS- $\alpha_{1}$, we performed PCR using an OlGCS- $\alpha_{1}$ clone in pBluescript II KS(-) as a template. PCR was performed using Pfu polymerase (TaKaRa) according to the manufacturer's protocol with various primers (Table 1). The reaction parameters used for PCR were as follows: 30 cycles of $30 \mathrm{sec}$ at $94^{\circ} \mathrm{C}, 30 \mathrm{sec}$ at each annealing temperature shown in Table 1 , and $2 \mathrm{~min}$ at $72^{\circ} \mathrm{C}$, with a final extension of $5 \mathrm{~min}$ at $72^{\circ} \mathrm{C}$. Each PCR product was purified and digested by SpeI and XhoI. Resulting SpeI/XhoI cut PCR product was subcloned into pFASTBAC1 vector.

\section{Construction of plasmids for C-terminal FLAG-tagged site-directed OIGCS- $\alpha_{1}$ mutant proteins}

Mutagenesis of OlGCS- $\alpha_{1}$ was performed using the following primers: 5'-GCC

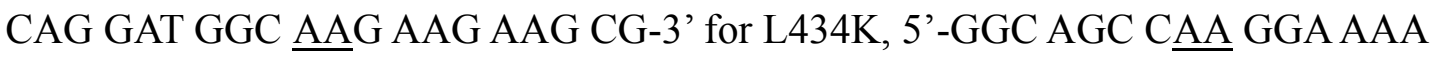
CGC TCA CC-3' for L445K, 5'-CGC TCA CCA AGC GAA GGA GGA GG-3' for

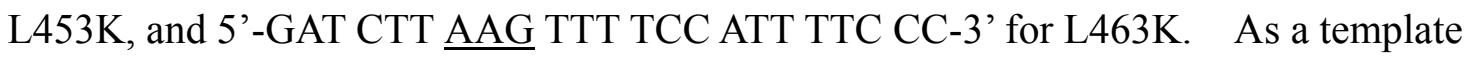
for the mutagenesis, we used the pFASTBAC1 vector containing C-terminal FLAG-tagged intact OIGCS- $\alpha_{1}$-full length sequence. The mutagenesis was performed 
by the "site-directed and semi-random mutagenesis" procedure (Sawano and Miyawaki, 2000).

\section{Generation of recombinant baculovirus}

All clones were checked for correctness of the sequence by DNA sequencing. The sequence was determined by the dideoxy chain termination procedure (Sanger et al., 1992) with an ABI PRISM 3100 Genetic Analyzer (Applied Biosystems, Osaka, Japan) and analyzed with GENETYX-MAC/version 7.2.0 software (Software Development, Tokyo, Japan). Recombinant baculovirus of the respective clone was generated according to a BAC-TO-BAC Baculovirus Expression System (Invitrogen Japan K. K.).

\section{Sf9 cell culture, expression of recombinant soluble GC subunit constructs and the mutants, and cytosol preparation}

Sf9 cells were cultured in SF-900 II serum-free medium supplemented with 50 $\mu \mathrm{g} / \mathrm{ml}$ streptomycin, $50 \mathrm{U} / \mathrm{ml}$ penicillin, and $20 \%$ fetal bovine serum (FBS). Spinner cultures were grown to a cell density of $3.0 \times 10^{6}$ cells $/ \mathrm{ml}$ and then diluted to $2.0 \times 10^{6}$ cells $/ \mathrm{ml}$ in a tissue culture flask for infection. Ten $\mathrm{ml}$ of the culture medium

containing Sf9 cells $\left(2.0 \times 10^{6}\right.$ cells $\left./ \mathrm{ml}\right)$ were infected by each virus stock of OlGCS- $\alpha_{1}$ or co-infected with OlGCS- $\beta_{1}-\mathrm{FL}_{\mathrm{HT}}$-containing virus (ratio $1: 1$ ), and continued to culture for 4 days at $37^{\circ} \mathrm{C}$. All subsequent experiments were carried out at $4^{\circ} \mathrm{C}$. Cells were harvested by centrifugation at $1,000 \mathrm{~g}$ for $5 \mathrm{~min}$. The resulting pellet was washed in $1 \mathrm{ml}$ of ice-cold phosphate-buffered saline (PBS), $\mathrm{pH}$ 6.2, and then resuspended in $500 \mu \mathrm{l}$ of an ice-cold lysis buffer for pull down assay (50 mM phosphate buffer, $\mathrm{pH} 7.2$ containing $100 \mathrm{mM} \mathrm{NaCl}, 10 \%$ glycerol, and $0.1 \%$ protease inhibitor mixture in DMSO solution) (Wako, Osaka, Japan) or a lysis buffer for assay of GC activity (20 mM 
Tris-HCl, pH 6.8, $90 \mathrm{mM} \mathrm{NaCl}, 10 \%$ glycerol, and $0.1 \%$ protease inhibitor mixture-dimethylsulfoxide (DMSO) solution). The suspension was sonicated and then centrifuged at 15,000 g for $20 \mathrm{~min}$, and the resulting supernatant fraction was collected and used for further experiments.

\section{Determination of protein concentration, Western blotting, and pull down assay}

Protein concentration was determined by the BCA method using BCA protein assay reagent (PIERCE, Rockford, Illinois, USA). Before each pull down assay, the similar expression level of the protein in each lysate was confirmed by sodium dodecyl sulfate-polyacrylamide gel electrophoresis (SDS-PAGE) and Western blotting. The cell lysate was subjected to SDS-PAGE on a $10 \%$ polyacrylamide gel and transferred to a PVDF membrane. The membrane was blocked with $2 \%$ dry milk in TPBS (PBS, pH 7.4, containing $0.1 \%$ Tween 20 ) for $15 \mathrm{~min}$ at room temperature, rinsed, and incubated overnight at $4^{\circ} \mathrm{C}$ with anti-His-Tag antibody (MBL, Nagoya, Japan) or ANTI-FLAG M2 monoclonal antibody-alkaline phosphatase conjugate (SIGMA, Saint Louis, Missouri, USA) in TPBS containing $0.2 \%$ dry milk. Subsequently, the anti-His-Tag antibody-treated membrane was incubated with secondary antibody for $1 \mathrm{~h}$ at room temperature. Immunoreacted proteins were detected using the CDP-Star, ready-to-use (Roche, Tokyo, Japan) or Western blotting detection reagents (Amersham). The density of each band was determined by scanned image using Scion Image Beta 4.0.2 (Scion corporation, Frederick, Maryland, USA). The lysis buffer for pull down assay containing $500 \mu \mathrm{g}$ protein was incubated overnight at $4^{\circ} \mathrm{C}$ with $150 \mu 1$ of chelating Sepharose (Amersham Pharmacia Biotech, Tokyo, Japan), which was preincubated in a total volume of $600 \mu \mathrm{l}$ containing $75 \mu \mathrm{l}$ of $0.2 \mathrm{M} \mathrm{NiCl}_{2}$ for $5 \mathrm{~min}$ according to the manufacture's protocol. The sample was washed 3 times with $750 \mu 1$ of the ice-cold 
lysis buffer for pull down assay, and then washed 3 times with $300 \mu 1$ of the ice-cold lysis buffer for pull down assay containing $15 \mathrm{mM}$ imidazole. Proteins were eluted with the ice-cold lysis buffer for pull down assay containing $150 \mathrm{mM}$ imidazole. Eluted proteins were subjected to SDS-PAGE and Western blotting as described above.

\section{Assay of GC activity}

Before the assay of GC activity, the similar expression level of the protein in each cytosolic fraction was confirmed by SDS-PAGE and Western blotting. GC activity of the cytosolic fraction (50 $\mu \mathrm{g}$ protein/assay tube) was determined in a total volume of $250 \mu \mathrm{l}$ by incubation for $15 \mathrm{~min}$ at $37^{\circ} \mathrm{C}$ in an assay solution $(50 \mathrm{mM}$ Tris- $\mathrm{HCl}, \mathrm{pH} 7.5$, $4 \mathrm{mM} \mathrm{MgCl} 2,0.5 \mathrm{mM}$ 3-isobutyl-1-methylxanthine (IBMX), $7.5 \mathrm{mM}$ creatine phosphate, $25 \mathrm{U} / \mathrm{ml}$ creatine phosphokinase, and $1 \mathrm{mM} \mathrm{GTP}$ ) with or without $1 \mathrm{mM}$ sodium nitroprusside dihydrate (SNP). The reaction was stopped by boiling for $3 \mathrm{~min}$, and then each assay tube was centifugated at $15,000 \mathrm{~g}$ for $10 \mathrm{~min}$. cGMP in the supernatant was determined by cGMP enzyme immunoassay system (Amersham Biosciences, Tokyo, Japan).

\section{RESULTS AND DISCUSSION}

\section{Identification of N-terminal binding site (NBS) and C-terminal binding site (CBS) of OIGCS- $\alpha_{1}$}

To assign the putative NBS- and CBS-like sequences of OlGCS- $\alpha_{1}$, the amino acid sequence of OlGCS- $\alpha_{1}$ was aligned with that of rat soluble GC- $\alpha_{1}$. Sequence identities of the NBS-like sequence of OlGCS- $\alpha_{1}$ at positions 280-321 and the CBS-like 
sequence of OlGCS- $\alpha_{1}$ at position 448-477 to that of the corresponding rat soluble GC- $\alpha_{1}$ were 45.2 and $90 \%$, respectively. To define the binding ability of the NBS-like sequence of OlGCS- $\alpha_{1}$ or the CBS-like sequence of OlGCS- $\alpha_{1}$ to OlGCS- $\beta_{1}$, we generated FLAG-tagged N-terminal deletion mutants or C-terminal deletion mutants of OlGCS- $\alpha_{1}$ (Fig. 1), and either one was expressed or co-expressed with the OlGCS- $\beta_{1}-\mathrm{FL}_{\mathrm{HT}}$ in Sf9 cells.

To examine the binding ability of the NBS-like sequence to OlGCS- $\beta_{1}-\mathrm{FL}_{\mathrm{HT}}$, we performed pull down assay (Fig. 2A). OlGCS- $\alpha_{1}-$ FL $_{\mathrm{FLAG}}, \mathrm{OlGCS}-\alpha_{1}[280-678]_{\mathrm{FLAG}}$ or OlGCS- $\alpha_{1}[322-678]_{\text {FLAG }}$ did not bind to $\mathrm{Ni}^{2+}$ Sepharose when each construct was expressed without OIGCS- $\beta_{1}-F L_{H T} . \quad$ Both OlGCS- $\alpha_{1}[280-678]_{\mathrm{FLAG}}$ and OlGCS- $\alpha_{1}[322-678]_{\mathrm{FLAG}}$ fully retained their ability to bind to OlGCS- $\beta_{1}-\mathrm{FL}_{\mathrm{HT}}$, judging from the result that each OlGCS- $\alpha_{1} \mathrm{~N}$-terminal deletion protein was detected in the $\mathrm{Ni}^{2+}$ Sepharose-binding fraction. This suggests that the NBS-like sequence of OlGCS- $\alpha_{1}$ (280-321) is not involved in the heterodimerization with OlGCS- $\beta_{1}$.

We then examined the binding ability of the $\mathrm{C}$-terminal deletion mutant proteins of OlGCS- $\alpha_{1}$ to OlGCS- $\beta_{1}$. Co-expression of OlGCS $-\alpha_{1}[1-448]_{F L A G}$ or OlGCS- $\alpha_{1}[1-477]_{F L A G}$ with OIGCS- $\beta_{1}-F L_{H T}$ was followed by pull down assay and Western blotting. As shown in Fig. 2B, OlGCS- $\alpha_{1}[1-448]_{\text {FLAG }}$ did not exhibit the binding ability to OlGCS- $\beta_{1}-\mathrm{FL}_{\mathrm{HT}}$, and no protein band was detected in the $\mathrm{Ni}^{2+}$ Sepharose-binding fraction of $\alpha_{1}[1-448]_{\mathrm{FLAG}} / \beta_{1}-\mathrm{FL}_{\mathrm{HT}}$ by anti-FLAG antibody. Conversely, OlGCS- $\alpha_{1}[1-477]_{\mathrm{FLAG}}$ bound to OlGCS $-\beta_{1}-\mathrm{FL}_{\mathrm{HT}}$, suggesting that the CBS-like sequence of OlGCS- $\alpha_{1}$ contains the structural elements mediating heterodimerization with OlGCS- $\beta_{1}-\mathrm{FL}_{\mathrm{HT}}$.

To examine the GC activity of the N-terminal deletion mutant proteins of OlGCS- $\alpha_{1}$, OlGCS- $\alpha_{1}-F L_{F L A G}$, OlGCS- $\alpha_{1}[280-678]_{F L A G}$ or OlGCS- $\alpha_{1}[322-678]_{F L A G}$ was 
co-expressed with OlGCS- $\beta_{1}-F L_{H T}$ in Sf9 cells and the GC activity of the each cytosolic fraction was measured. As shown in Fig. 3, $\alpha_{1}[280-678]_{\mathrm{FLAG}} / \beta_{1}-\mathrm{FL}_{\mathrm{HT}}$ showed the basal and SNP-stimulated GC activity at almost the same extent as that of the control soluble GC $\left(\alpha_{1}-\mathrm{FL}_{\mathrm{FLAG}} / \beta_{1}-\mathrm{FL}_{\mathrm{HT}}\right)$, although $\alpha_{1}-[322-678]_{\mathrm{FLAG}} / \beta_{1}-\mathrm{FL}_{\mathrm{HT}} \operatorname{did}$ not exhibit GC activity with and without SNP. Therefore, we presume that the NBS-like sequence of OlGCS- $\alpha_{1}$ (amino acids 280-321) plays an important role to form the correct active center with the counterpart. This is in good agreement with the results that the $\beta_{1}$ subunit lacking amino acids at position 204-303 (containing NBS: amino acids positions 204-244) failed to show the GC activity when co-expressed with the $\alpha_{1}$ subunit, although it exhibits the binding ability to the $\alpha_{1}$ subunit at a reduced level (Zhou et al., 2004). In a recent study, it was reported that an N-terminal binding site of bovine $\alpha_{1}$ subunit (amino acids position 61-128) plays major important role in heterodimerization (Wagner et al., 2005). However, in this study, we did not observed such ability in the amino acids position 61-128. In the present study with the pull down assay and the assay of GC activity, we obtained the results to show that OlGCS- $\alpha_{1}[280-678]_{\mathrm{FLAG}}$ could form heterodimer with OlGCS- $\beta_{1}-\mathrm{FL}_{\mathrm{HT}}$.

\section{Identification of amphipathic $\alpha$-helix mediating heterodimerization between OIGCS- $\alpha_{1}$ and OIGCS- $\beta_{1}$}

To obtain further understanding on the nature of the CBS-like sequence of OlGCS- $\alpha_{1}$ for heterodimerization and GC activity, we carried out prediction of the secondary structure of OlGCS- $\alpha_{1}$ by the PredictProtein server (http://www.embl-heidelberg.de/predictprotein/) and found that OlGCS- $\alpha_{1}[431-464]$ is able to form an amphipathic $\alpha$-helix structure (Fig. 4). It has been reported that amphipathic $\alpha$-helix often mediates protein-protein interactions (Carr et al, 1991). 
Therefore, we examined whether the amphipathic $\alpha$-helix in the $\alpha_{1}$-subunit could mediate heterodimerization of both subunits of soluble GC after introduction of site-directed mutation to the $\alpha_{1}$-subunit. To disturb the amphipathicity of the $\alpha$-helix, we focused the four Leu residues $\left(\mathrm{Leu}^{434}, \mathrm{Leu}^{445}, \mathrm{Leu}^{452}\right.$, and $\mathrm{Leu}^{463}$ ) as shown in dotted boxes in Fig. 4B, all of which were predicted to be located intensively on the one side of the $\alpha$-helix. Various site-directed mutant proteins were generated as described in Materials and Methods. According to the prediction of the secondary structure of the $\alpha_{1}$ subunit by the PredictProtein server, the $\alpha$-helix structure should be maintained even after these four Leu residues were converted to Lys residues leading to loss of amphipathicity. $\quad$ OlGCS- $\alpha_{1}-L 434 K_{F L A G}, L 445 K_{F L A G}, L 463 K_{F L A G}, L 434,445,463 K_{F L A G}$, or $L 434,445,452,463 K_{F L A G}$ was co-expressed with OlGCS- $\beta_{1}-F L_{H T}$ in Sf9 cells and the GC activity of the each cytosolic fraction was measured with or without SNP. As shown in Fig. $5, \alpha_{1}-\mathrm{L} 434 \mathrm{~K}_{\mathrm{FLAG}} / \beta_{1}-\mathrm{FL}_{\mathrm{HT}}$ or $\alpha_{1}-\mathrm{L} 445 \mathrm{~K}_{\mathrm{FLAG}} / \beta_{1}-\mathrm{FL}_{\mathrm{HT}}$ showed the basal and SNP-stimulated GC activities at almost the same extent as those of $\alpha_{1}-\mathrm{FL}_{\mathrm{FLAG}} / \beta_{1}-\mathrm{FL}_{\mathrm{HT}}$, but $\alpha_{1}-\mathrm{L}_{4} 63 \mathrm{~K}_{\mathrm{FLAG}} / \beta_{1}-\mathrm{FL}_{\mathrm{HT}}, \mathrm{L} 434,445,463 \mathrm{~K}_{\mathrm{FLAG}} / \beta_{1}-\mathrm{FL}_{\mathrm{HT}}$ or $\mathrm{L} 434,445,452,463 \mathrm{~K}_{\mathrm{FLAG}} / \beta_{1}-\mathrm{FL}_{\mathrm{HT}}$ did not show the basal GC activity nor the SNP-stimulatable GC activity. To investigate the binding ability of each site-directed mutant protein to OlGCS- $\beta_{1}-\mathrm{FL}_{\mathrm{HT}}$, we performed pull down assay. As shown in Fig. 6, the binding ability of OlGCS- $\alpha_{1}-\mathrm{L}_{4} 434,445,463 \mathrm{~K}_{\mathrm{FLAG}}$ or OlGCS- $\alpha_{1}-\mathrm{L} 434,445,452,463 \mathrm{~K}_{\mathrm{FLAG}}$ to OlGCS- $\beta_{1}-\mathrm{FL}_{\mathrm{HT}}$ was remarkably reduced, although $\alpha_{1}-\mathrm{L}_{4} 43 \mathrm{~K}_{\mathrm{FLAG}}$ or $\alpha_{1}-\mathrm{L}_{4} 45 \mathrm{~K}_{\mathrm{FLAG}}$ maintained its binding ability to OlGCS- $\beta_{1}-\mathrm{FL}_{\mathrm{HT}}$. These results suggest that the amphipathicity in the putative $\alpha$-helix region of OlGCS- $\alpha_{1}$ is important to form heterodimer with OlGCS- $\beta_{1}$.

The amphipathicity of the $\alpha$-helix in OlGCS- $\alpha_{1}[1-448]_{\text {FLAG }}$ would be incomplete because of lacking the C-terminal half of the putative amphipathic $\alpha$-helix (see Fig 1). 
Therefore, we presume that it is one of the major reasons why OlGCS- $\alpha_{1}[1-448]_{\text {FLAG }}$ did not exhibit the binding ability to OlGCS $-\beta_{1}$. Surprisingly, OlGCS- $\alpha_{1}$-L463K $\mathrm{FLAG}_{\mathrm{F}}$ also maintained its binding ability to OlGCS- $\beta_{1}-\mathrm{FL}_{\mathrm{HT}}$, although the $\alpha_{1^{-}}$ L463K $\mathrm{K}_{\mathrm{FLAG}} / \beta_{1}-\mathrm{FL}_{\mathrm{HT}}$ did not exhibit any GC activity, suggesting that the formation of heterodimer of OlGCS- $\alpha_{1}$ with OlGCS- $\beta_{1}$ is not sufficient for exhibiting the GC activity, probably due to inadequate heterodimerization which leads to formation of inadequate active center. As shown in Fig. 4A, Leu ${ }^{463}$ is conserved among soluble GC- $\alpha_{1}$ subunits of various species and located in the C-terminal end of the amphipathic $\alpha$-helix. For enzymatically active heterodimer formation, it seems to be essential that the correct active center between the catalytic domains of $\alpha$ and $\beta$ subunit is formed. Conversion of Leu ${ }^{463}$ to Lys in OlGCS- $\alpha_{1}$ might lead the detachment of the hydrophobic bond in the C-terminal end of the amphipathic $\alpha$-helix, although the rest of the amphipathic $\alpha$-helix maintains the hydrophobic bonds. The detachment of this region appears to lead to the incorrect active center formation between the both subunit catalytic domains. This might be the case that $\mathrm{L} 463 \mathrm{~K}_{\mathrm{FLAG}} / \beta_{1}-\mathrm{FL}_{\mathrm{HT}}$ did not show any GC activity.

As shown in Fig. 4A, the amino acid sequence in this region varies among soluble GC $\alpha_{1}$ subunits of various species and the changes in the amino acid sequence did not affect the formation of the putative amphipathic $\alpha$-helix, suggesting that the amphipathic $\alpha$-helix plays a critical role in the heterodimerization of soluble GC $\alpha_{1}$ subunit with soluble GC $\beta_{1}$ subunit. In addition, the analysis of the secondary structure of OlGCS- $\beta_{1}$ by the PredictProtein server predicted that OlGCS- $\beta_{1}$ [367-395] forms an amphipathic $\alpha$-helix, and similar analysis of OlGCS- $\alpha_{2}$ also resulted in prediction that OlGCS- $\alpha_{2}[535-555]$ forms an amphipathic $\alpha$-helix. These strongly suggest that the amphipathic $\alpha$-helix mediates the heterodimerization of soluble GC $\alpha_{1}$ subunit with soluble GC $\beta_{1}$ subunits. 


\section{ACKNOWLEGEMENTS}

We are grateful to the staff members of the Center for Advanced Science and Technology, Hokkaido University, for the use of their laboratory facilities. This study was supported by a Grant-in-Aid for Scientific Research from the Ministry of Education, Science, Sports, and Culture of Japan (no. 11236202) and by the National Project on Protein Structural and Functional Analyses. 


\section{REFERENCES}

Carr DW, Stofko-hahn RE, Fraser ID, Bishop SM, Acott TS, Brennan RG, Scott JD (1991) Interaction of the regulatory subunit (RII) of cAMP-dependent protein kinase with RII-anchoring proteins occurs through an amphipathic helix binding motif. J Biol Chem 266: 14188-14192

Chomczynski P, Sacchi N (1987) Single-step method of RNA isolation by acid guanidinium thiocyanate-phenol-chloroform extraction. Anal Biochem 162: $156-159$

Drewett JG, Garbers DL (1994) The family of guanylyl cyclase receptors and their ligands. Endocr Rev 15: 135-162

Foerster J, Harteneck C, Malkewitz J, Schultz G, Koesling D (1996) A functional heme-binding site of soluble guanylyl cyclase requires intact N-termini of $\alpha_{1}$ and $\beta_{1}$ subunits. Eur J Biochem 240: 380-386

Garbers DL, Koesling D, Schultz G (1994) Guanylyl cyclase receptors. Mol Biol Cell 5: $1-5$

Garbers DL, Lowe DG (1994) Guanylyl cyclase receptors. J Biol Chem 269: $30741-30744$

Gerzer R, Böhme E, Hofmann F, Schultz G (1981) Soluble guanylate cyclase purified from bovine lung contains heme and copper. FEBS Lett 132: 71-74

Harteneck C, Wedel B, Koesling D, Malkewitz J, Böhme E, Schultz G (1991)

Molecular cloning and expression of a new alpha-subunit of soluble guanylyl cyclase. Interchangeability of the alpha-subunits of the enzyme. FEBS Lett 292: $217-222$

Humbert P, Niroomand F, Fischer G, Mayer B, Koesling D, Hinsch KD, Gausepohl H, 
Frank R, Schultz G, Böhme E (1990) Purification of soluble guanylyl cyclase from bovine lung by a new immunoaffinity chromatographic method. Eur J Biochem 190: $273-278$

Koesling D (1999) Studying the structure and regulation of soluble guanylyl cyclase. Methods 19: 485-493

Koesling D, Herz J, Gausepohl H, Niroomand F, Hinsch KD, Mulsch A, Böhme E, Schultz G, Frank R (1988) The primary structure of the $70 \mathrm{kDa}$ subunit of bovine soluble guanylate cyclase. FEBS Lett 239: 29-34

Koglin M, Behrends S (2003) A functional domain of the $\alpha 1$ subunit of soluble guanylyl cyclase is necessary for activation of the enzyme by nitric oxide and YC-1 but is not involved in heme binding. J Biol Chem 278: 12590-12597

Mikami T, Kusakabe T, Suzuki N (1998) Molecular cloning of cDNAs and expression of mRNAs encoding alpha and beta subunits of soluble guanylyl cyclase from medaka fish Oryzias latipes. Eur J Biochem 253: 42-48

Mikami T, Kusakabe T, Suzuki N (1999) Tandem organization of medaka fish soluble guanylyl cyclase $\alpha_{1}$ and $\beta_{1}$ subunit genes. Implications for coordinated transcription of two subunit genes. J Biol Chem 274: 18567-18573

Russwurm M, Behrends S, Harteneck C, Koesling D (1998) Functional properties of a naturally occurring isoform of soluble guanylyl cyclase. Biochem J 335: 125-130

Sanger F, Nicklen S, Coulson AR (1992) DNA sequencing with chain-terminating inhibitors. 1977. Biotechnology 24: 104-108

Sawano A, Miyawaki A (2000) Directed evolution of green fluorescent protein by a new versatile PCR strategy for site-directed and semi-random mutagenesis. Nucleic Acids Res 28: E78.

Stone JR, Marletta MA (1994) Soluble guanylate cyclase from bovine lung: activation 
with nitric oxide and carbon monoxide and spectral characterization of the ferrous and ferric state. Biochemistry 33: 5636-5640

Sunahara RK, Beuve A, Tesmer JJ, Sprang SR, Garbers DL, Gilman AG (1998) Exchange of substrate and inhibitor specificities between adenylyl and guanylyl cyclase. J Biol Chem 273: 16332-16338

Tesmer JJ, Sunahara RK, Gilman AG, Sprang SR (1997) Crystal structure of the catalytic domains of adenylyl cyclase in a complex with Gs $\alpha$ GTP $\gamma$ S. Science 278: 1907-1916

Tucker CL, Hurley JH, Miller TR, Hurley JB (1998) Two amino acid substitutions convert a guanylyl cyclase, Ret GC-1, into an adenylyl cyclase. Proc Natl Acad Sci USA 95: 5993-5997

Wagner C, Russwurm M, Jäger R, Friebe A, Koesling D (2005) Dimerizaion of NO-sensitive guanylyl cyclase requires the $\alpha_{1} \mathrm{~N}$ terminus. J Biol Chem 280:17687-17693

Wedel B, Harteneck C, Foerster J, Friebe A, Schultz G, Koesling D (1995) Functional domains of soluble guanylyl cyclase. J Biol Chem 270: 24871-24875

Wedel B, Humbert P, Harteneck C, Foerster J, Malkewitz J, Böhme E, Schultz G, Koesling D (1994) Mutation of His-105 in the $\beta_{1}$ subunit yields a nitric oxide-insensitive form of soluble guanylyl cyclase. Proc Natl Acad Sci USA 91: 2592-2596

Wilson EM, Chinkers M (1995) Identification of sequences mediating guanylyl cyclase dimerization. Biochemistry 34: 4696-4701

Yao Y, Yamamoto T, Tsutsumi M, Matsuda M, Hori H, Naruse K, Mitani H, Shima A, Asakawa S, Shimizu N, Suzuki N (2003) Genomic structure and expression of the soluble guanylyl cyclase $\alpha_{2}$ subunit gene in the medaka fish Oryzias latipes. Zool 
Sci 20: 1293-1304

Yuen PS, Potter LR, Garbers DL (1990) A new form of guanylyl cyclase is preferentially expressed in rat kidney. Biochemistry 29: 10872-10878

Zabel U, Hausler C, Weeger M, Schmidt HH (1999) Homodimerization of soluble guanylyl cyclase subunits. Dimerization analysis using a glutathione s-transferase affinity tag. J Biol Chem 274: 18149-18152

Zhang G, Liu Y, Ruoho AE, Hurley JH (1997) Structure of the adenylyl cyclase catalytic core. Nature 386: 247-253

Zhao Y, Schelvis JP, Babcock GT, Marletta MA (1998) Identification of histidine 105 in the $\beta_{1}$ subunit of soluble guanylate cyclase as the heme proximal ligand. Biochemistry 37: 4502-4509

Zhou Z, Gross S, Roussos C, Meurer S, Müller-Esterl W, Papapetropoulos A (2004) Structural and functional characterization of the dimerization region of soluble guanylyl cyclase J Biol Chem 279: 24935-24943 


\section{Legends to Figures}

Fig. 1. Schematic representation of various OlGCS- $\alpha_{1}$ constructs.

The NBS-like sequence (280-321) is represented by the hatched boxes and the CBS-like sequence is defined by dotted boxes. Numbers denote the relative positions of amino acids. A bold line indicates the predicted amphipathic $\alpha$-helix region.

Fig.2. Pull down assay on the NBS-like or the CBS-like sequence.

Sf9 cells were infected by baculovirus containing an OIGCS- $\alpha_{1}-F L_{F L A G}$, its N-terminal deletion mutants (A) or its C-terminal deletion mutants (B) with or without

OlGCS- $\beta_{1}-F L_{H T} . \quad$ Almost equal amount of OlGCS- $\beta_{1}-\mathrm{FL}_{\mathrm{HT}}$ was detected in the $\mathrm{Ni}^{2+}$ Sepharose-binding fraction by Western blotting (WB) using anti-HT antibody (top).

The $\mathrm{Ni}^{2+}$ Sepharose-binding fraction was analyzed by Western blotting with anti-FLAG antibody (upper middle). To confirm the protein expression levels in the lysate, Western blotting was performed using anti-HT antibody or anti-FLAG antibody (lower middle and bottom, respectively). Similar results were obtained in two independent experiments.

Fig. 3. The GC activity of N-terminal deletion mutant proteins.

The GC activity in the lysate prepared from respective infected Sf9 cells was assayed with (black columns) or without (white columns) SNP. The activity represent means $\underline{ \pm}$ S.E.M. obtained from three independent experiments.

Fig. 4. The sequence and helical wheel diagram of the amphipathic $\alpha$-helix of OlGCS- $\alpha_{1}$. 
(A) The amino acid sequences of the putative amphipathic $\alpha$-helix of various soluble GC $\alpha_{1}$ subunits were aligned. Identical residues are marked by asterisks and the hydrophobic residues were shaded. Numbers denote the relative positions of the amino acids. (B) Hydrophobic amino acid residues are boxed and the Leu residues modified by site-directed mutagenesis are covered with dotted boxes.

Fig. 5. The GC activity of site-directed mutant proteins.

The GC activity in the lysate prepared from respective infected Sf9 cells was assayed with (black columns) or without (white columns) SNP. The activity represents means \pm S.E.M. obtained from three independent experiments.

Fig. 6. Pull down assay of site-directed mutant proteins.

Sf9 cells were infected by baculovirus containing OlGCS- $\alpha_{1}-F L_{F L A G}$ or its site-directed mutants with OlGCS- $\beta_{1}-F L_{H T}$. The fractions containing the $\mathrm{Ni}^{2+}$ Sepharose-binding activity were analyzed by Western blotting with anti-FLAG antibody. The density of each band of the $\mathrm{Ni}^{2+}$ Sepharose-binding proteins reacted with anti-FLAG antibody was estimated using Scion image. The data are expressed by fold density relative to the $\alpha_{1}-\mathrm{FL}_{\mathrm{FLAG}} / \beta_{1}-\mathrm{FL}_{\mathrm{HT}}$ as 1 and represent means \pm S.E.M. obtained from three independent experiments. $\quad * \mathrm{P}<0.05$ for the comparison with $\alpha_{1}-\mathrm{L} 434 \mathrm{~K}_{\mathrm{FLAG}} / \beta_{1}-\mathrm{FL}_{\mathrm{HT}}$ or $\alpha_{1}-\mathrm{L}_{4} 45 \mathrm{~K}_{\mathrm{FLAG}} / \beta_{1}-\mathrm{FL}_{\mathrm{HT}}$. 
Table1 Primers and the annealing temperature used for construction of plasmids for OlGCS- $\alpha_{1}-\mathrm{FL}_{\mathrm{FLAG}}$ and its truncated mutants 
Sequence of primers

Annealing

temperature

a1-FLFLAG

$44^{\circ} \mathrm{C}$

a1start-SpeI 5'-ACTAGTATGTTCTGCGGCCAAGTTGAA-3'

alendFLAG*XhoI 5'-CTCGAGTCACTTGTCATCGTCGTCCTTGTAGTCTTTTTTTGTAAGTTTTGACA-3'

a1[280-678]FLAG

$44^{\circ} \mathrm{C}$

a1-dN279 5'-ACTAGTATGACCTCTGCTGGAACGCTCCC-3'

alendFLAG*XhoI 5'-CTCGAGTCACTTGTCATCGTCGTCCTTGTAGTCTTTTTTTGTAAGTTTTGACA-3'

a1[322-678]FLAG

a1-dN321

5'-ACTAGTATGGGACTTAGAAGGTCTCCCAC-3'

$44^{\circ} \mathrm{C}$

alendFLAG*XhoI 5'-CTCGAGTCACTTGTCATCGTCGTCCTTGTAGTCTTTTTTTGTAAGTTTTGACA-3'

a1[1-477]

a1start-SpeI 5'-ACTAGTATGTTCTGCGGCCAAGTTGAA-3'

$54^{\circ} \mathrm{C}$

a1-dC477 5'-CTCGAGTCACTTGTCATCGTCGTCCTTGTAGTCCTGCCACAGCTGCTGGGCCA-3'

a1 $[1-448]$

$54^{\circ} \mathrm{C}$

a1start-SpeI 5'-ACTAGTATGTTCTGCGGCCAAGTTGAA-3'

a1-dC448 5'-CTCGAGTCACTTGTCATCGTCGTCCTTGTAGTCGTTTTCCAAGGCTGCCTTGG-3' 
Fig. 1

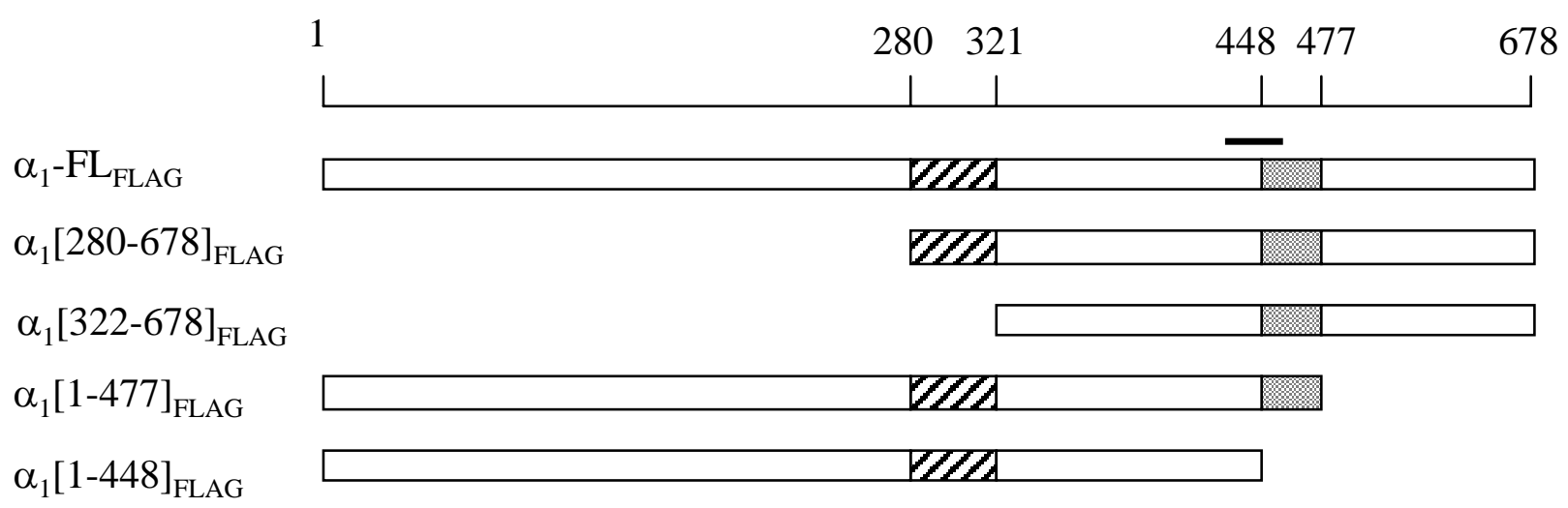


(A)

(B)

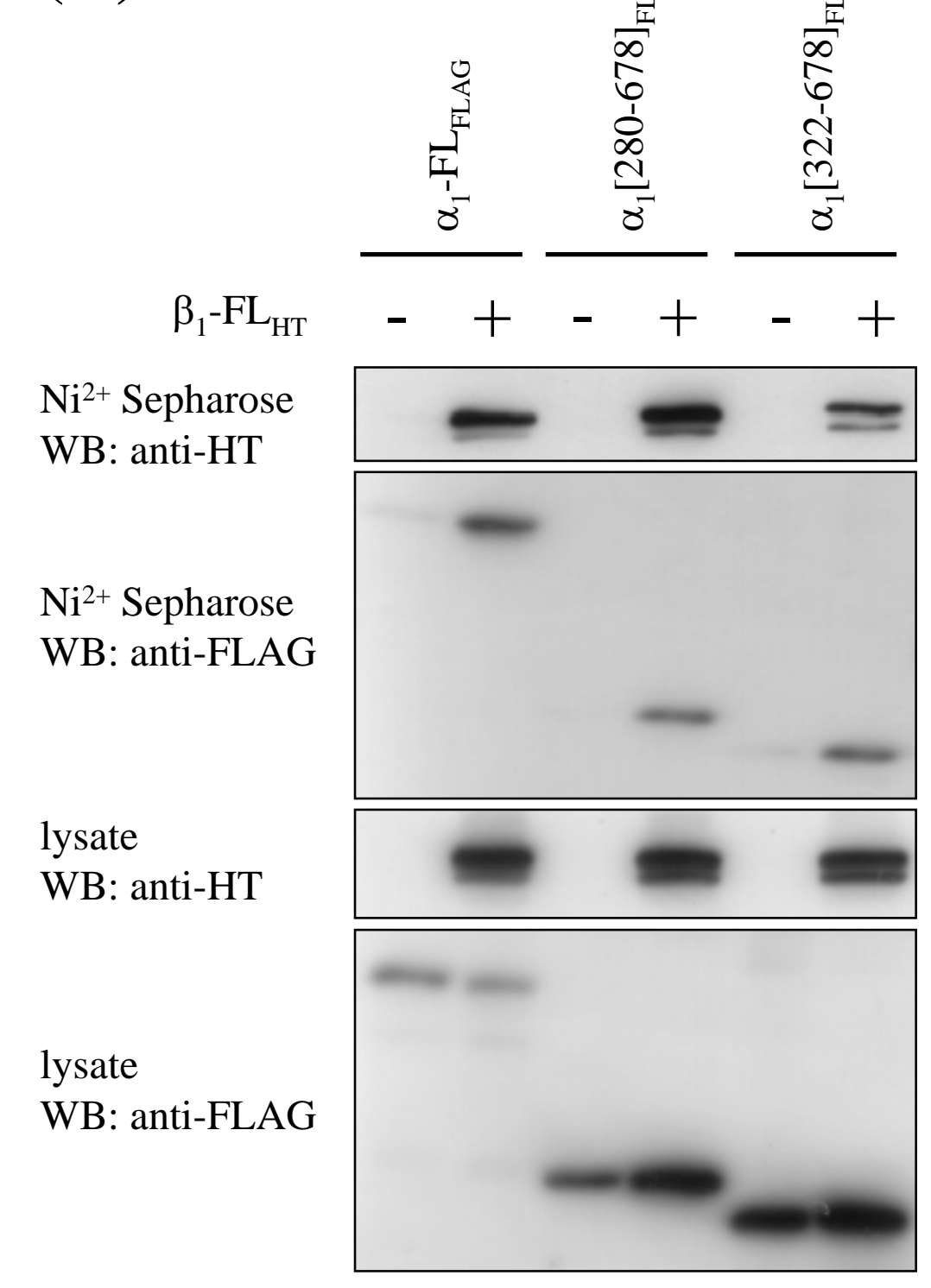

$\mathrm{Ni}^{2+}$ Sepharose

WB: anti-HT

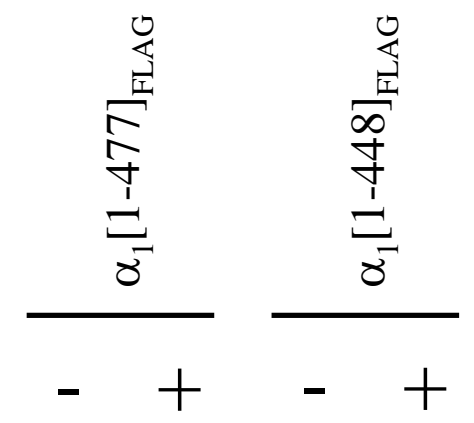

$\mathrm{Ni}^{2+}$ Sepharose

WB: anti-FLAG

lysate

WB: anti-HT

lysate

WB: anti-FLAG
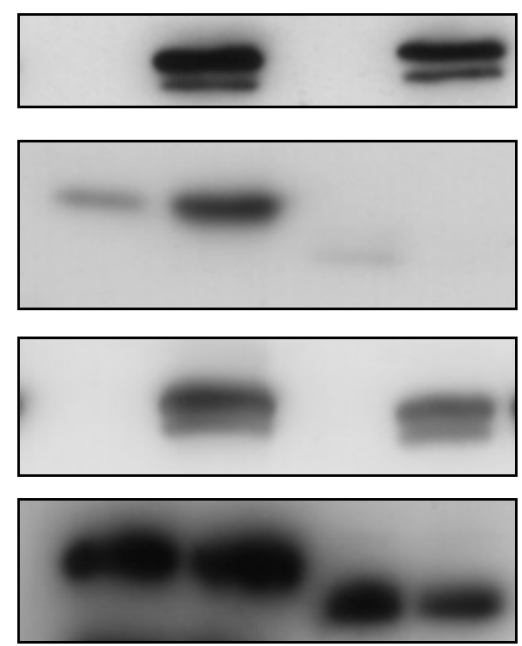
Fig. 3

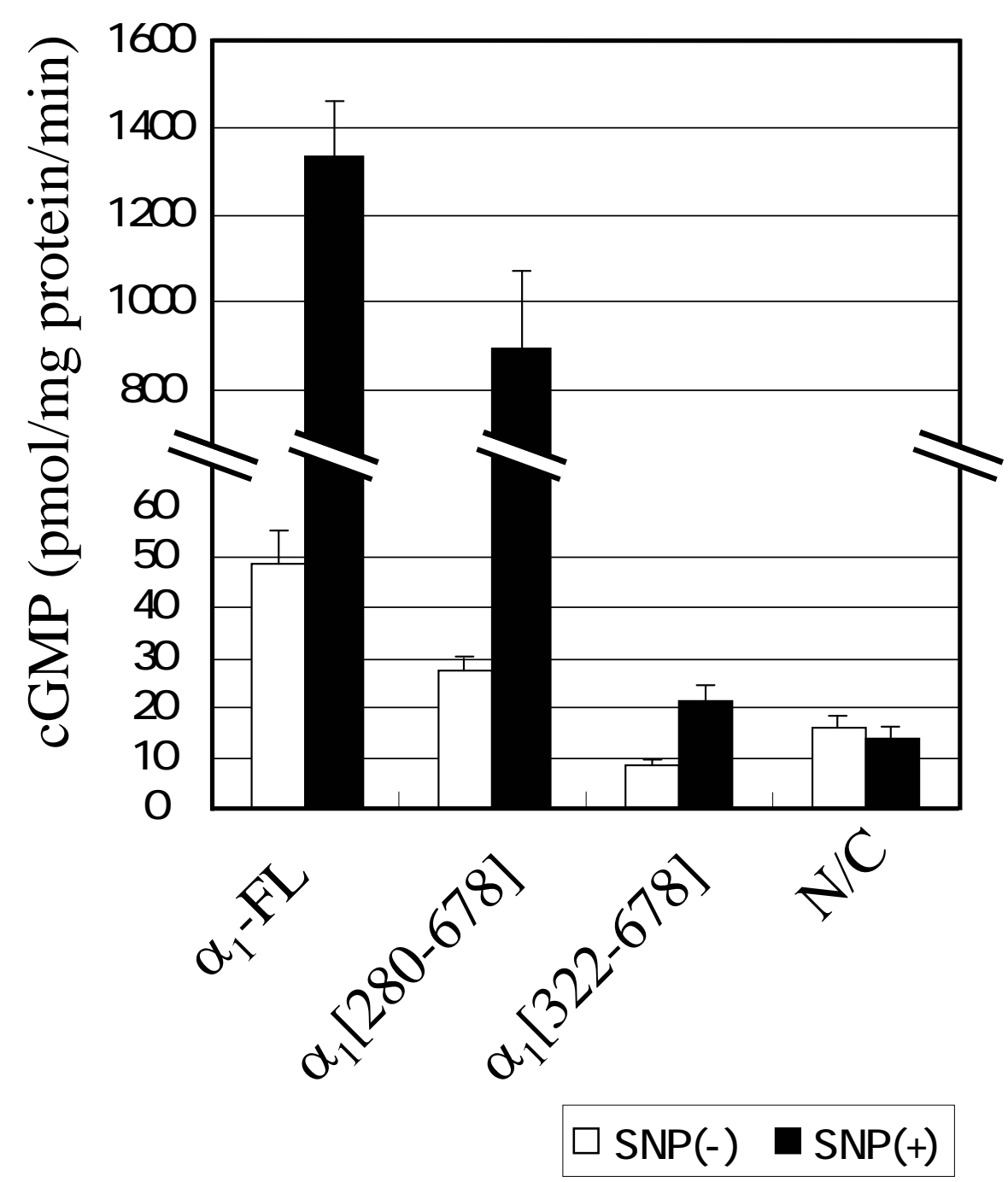




\section{(A)}

mouse

rat

human

medaka

Drosophila

Manduca
421 QDGLKKRLGKLKATLEHAHQALEEEKKRTVDLLC 455 420 QDGLKKRLGKLKATLEHAHQALEEEKKKTVDLLC 454 421 QDGLKKRLGKLKATLEQAHQALEEEKKKTVDLLC 455 431 QDGLKKRLGKAKAALENAHQALEEEKKKTVDLLF 464 406 QDGLRRRMDKIKNSIEEANSAVTKERKKNVSLLH 440 422 QDGLRRRMDKLKNSIEEASKAVDKEREKNVSLLH 456

\section{(B)}

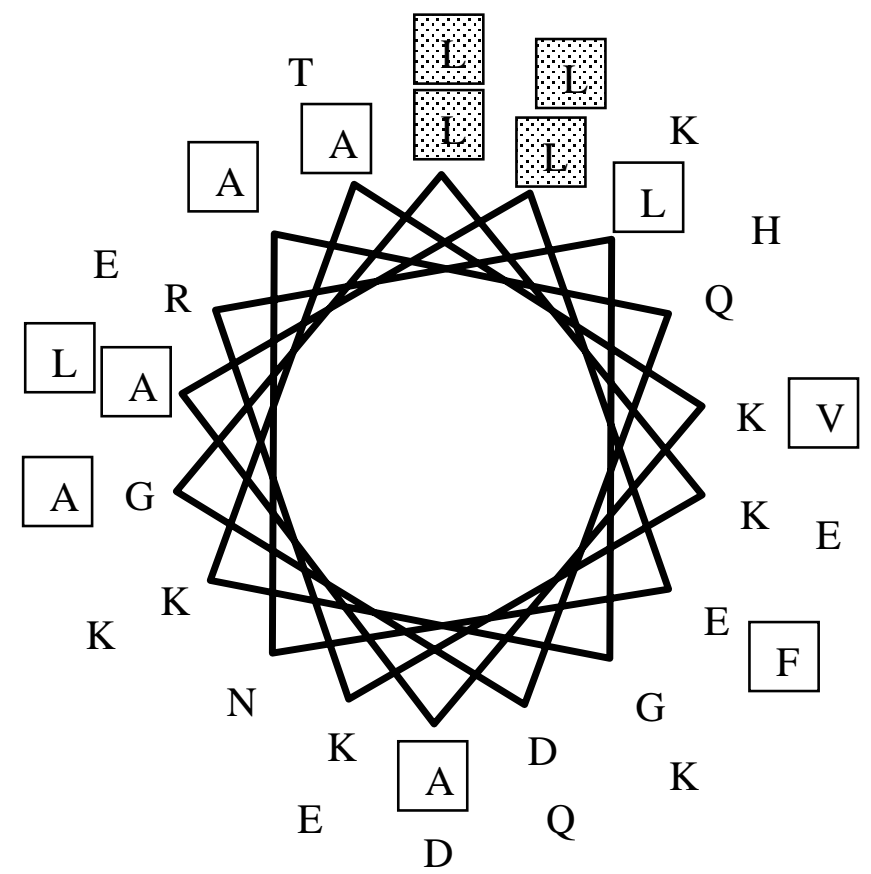


Fig. 5

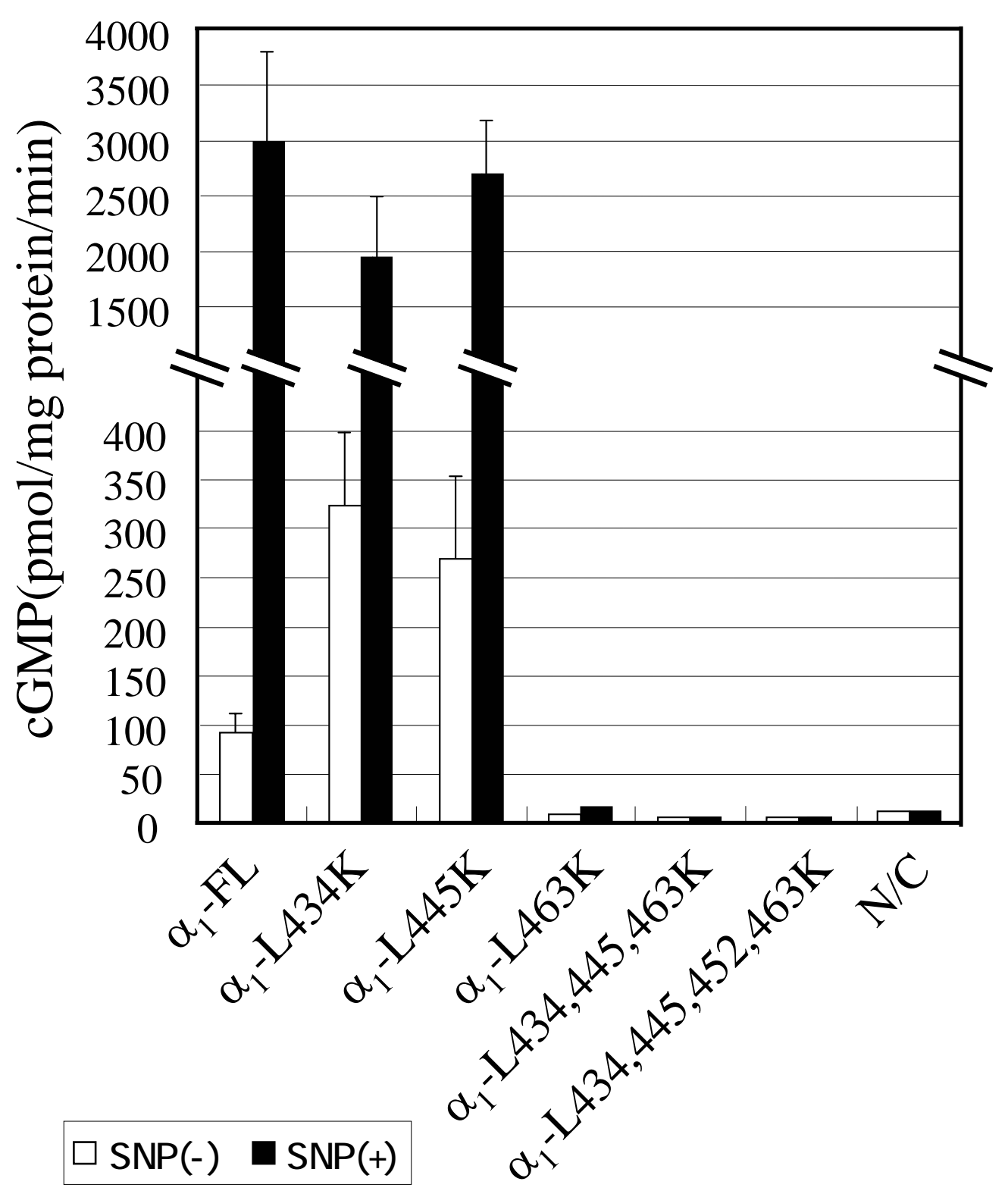


Fig. 6
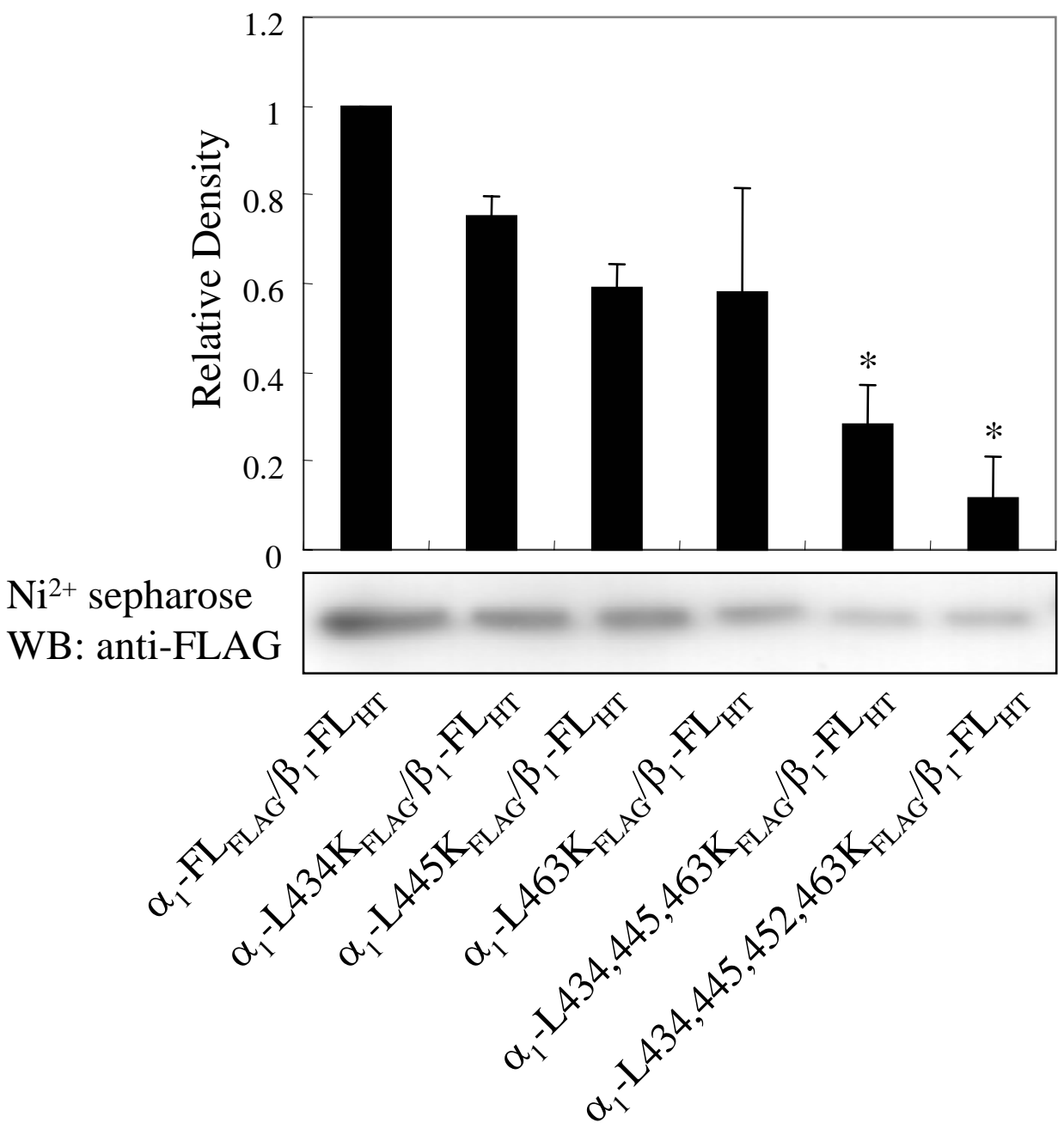